\title{
The dynamic of professional knowledge utilized in software applications for process controlling
}

\author{
Wladimir Bodrow
}

Received: 11 February 2014/ Accepted: 25 April 2014/Published online: 17 May 2014

(C) Shanghai University and Springer-Verlag Berlin Heidelberg 2014

\begin{abstract}
Main objective of the presented paper is the examination of the professional knowledge used in intelligent software applications for process controlling. It concerns in particular the user instruction and the realization of corresponding interaction as well as the interface design. Regular approach for service of the different user groups is evaluated and extended in means of flexibility in the time. The rules decisive for the user interaction with the application are outlined. They represent the interdependency between quality of professional knowledge to be utilized and user group characteristics alter in time. An application scenario for knowledge visualization is presented to show the practicability of the concept developed.
\end{abstract}

Keywords Intelligent controlling system - Levels of decision support · Professional knowledge · Process controlling

\section{Introduction}

At the very beginning of this paper some definitions of the business process are presented. The deep investigation and further development of the business process understanding is not the target of presented research therefore these will be given without comment.

Many authors with their different research orientations consider business process as a set of activities [1-5]. Instead of analyzing all available definitions made by different authors and trying to bring them to more or less

W. Bodrow ( $\square)$

Lab Innovative Systems, University of Applied Sciences HTW-

Berlin, Treskowallee, Berlin, Germany

e-mail: wladimir.bodrow@htw-berlin.de common understanding, we present next four different ones and consider them as an outgoing point of research presented in this paper.

According to the Appain-site, "A business process has also been defined as a set of activities and tasks that, once completed, will accomplish an organizational goal. The process must involve clearly defined inputs and a single output. These inputs are made up of all of the factors which contribute (either directly or indirectly) to the added value of a service or product. These factors can be categorized into management processes, operational processes and supporting processes". Rather compact definition one can find on the TechTarget-site (http://searchcio.techtarget. com/definition/business-process)_- “A business process is an activity or set of activities that will accomplish a specific organizational goal".

The definition given by Thomas Davenport is also focused on circumstances and attributes of single activities. He defines the business process as “... a structured, measured set of activities designed to produce a specific output for a particular customer or market. It implies a strong emphasis on how work is done within an organization, in contrast to a product focus's emphasis on what. A process is thus a specific ordering of work activities across time and space, with a beginning and an end, and clearly defined inputs and outputs: a structure for action" [6].

The characteristics of business process given in Wikipedia could be helpful to overview its complexity. Definability: it must have clearly defined boundaries, input and output. Order: it must consist of activities that are ordered according to their positions in time and space. Customer: there must be a recipient of the process' outcome, a customer. Value-adding: the transformation taking place within the process must add value to the recipient, either upstream or downstream. Embeddedness: a process cannot 
exist in itself and it must be embedded in an organizational structure. Cross-functionality: a process regularly can, but not necessarily must, span several functions.

In addition to the presented definitions, one can outline the factors of activity realization or execution. As listed below, enterprise resources influence the realization as single activity as the business process as a whole. They are not completed but essential for the further analysis. Human resources include professional knowledge, experience, etc. Material resources include finance resources, equipments (machines, materials, etc.), information and communication technology (ICT)-based resources (hardware, software, networks $\cdots)$. Comprehensive presentation of the topic models and instruments of human resources within the human resource management can be found in the book for instance written by Jens Rowold [7].

The focus in the following part of this paper will be the utilization of professional knowledge in business process. Especially it is related to ICT-based solutions and tools, e.g., knowledge-based systems, knowledge management systems, instruction systems, etc.

The structure under investigation in current research could be defined according to the scheme presented in Fig. 1.

The roles of involved elements and their influences on the realization of the single activity are presented in Fig. 2 .

The outgoing point for presented research is characterized by following aspects:

(i) Modern intelligent IT systems for process controlling in industry have to be flexible regarding the experience and professional knowledge of its users.

(ii) Generally such applications are not focused on absolute experts but on professionals with their individual skills and learning capabilities.

(iii) Developer of the application gets more and more inside knowledge during the project realization in the field and so it will be enabled to improve the system accordingly.

(iv) Certain amount of professional knowledge owned by system developers and integrated in the software relates to the quality of the application.

(v) Because of circumstances in particular process some controlling systems represent a set of ordered instructions according to the activities strictly to be fulfilled.

All mentioned above aspects are crucial for the efficient process controlling and hence for the implementation characteristics of corresponding IT systems. Design and realization of the user interface and interaction metaphor with application play a central role in this context $[8,9]$.

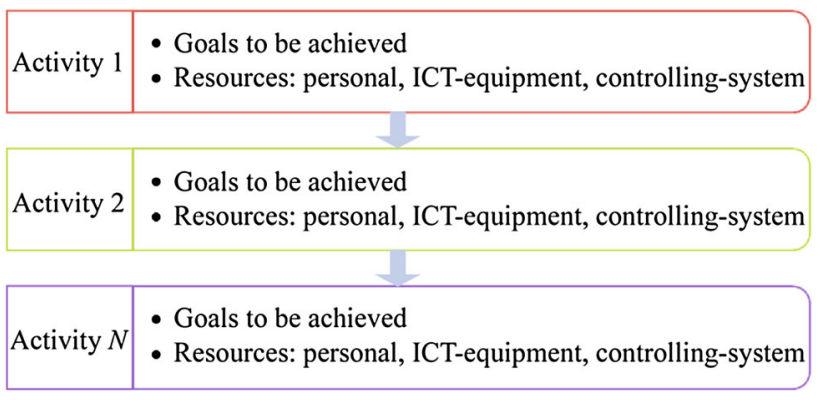

Fig. 1 Structure under investigation

Activity/task (e.g., manufacturing, design, etc.)

Controlling features (time, quality, cost, acceptance, etc.)

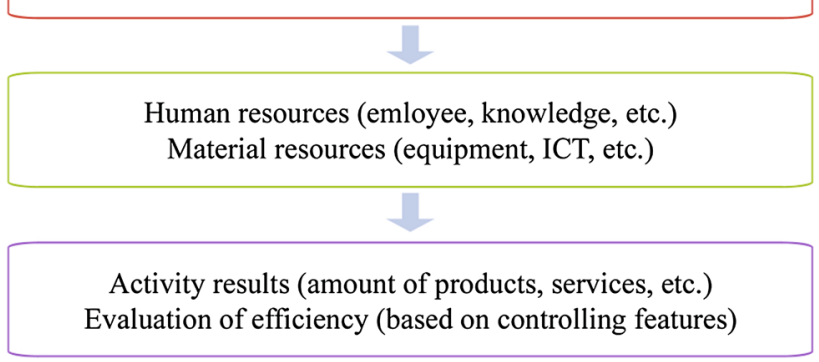

Fig. 2 Roles of elements in activity realization

Which knowledge is demanded and has to be visualized on the screen or presented on other device for Muller, Meyer, or Schulz today, tomorrow, in three months, or in two years? How it will be changed under given (changing) circumstances?

Following sections are devoted to the general features of knowledge dynamic to be used in software applications for process controlling. They are important for every individual solution, but do not represent one or another established (standardized) approach for development process of a single software application listed in Wikipedia.

\section{Professional knowledge of system users}

In this research the well-known Dreyfus model [10] of skill acquisition will be used in its reduced form. Three groups of users will be considered below:

(i) Novices are users with rather a little knowledge and experience in the field of application. They need the knowledge about the resources or elements involved in the process as well as about the interdependency between them and circumstances of the process controlling. 
(ii) Competent users possess knowledge about the process elements and are enabled to control the routine activities. They need support in nonregular situation, where they do not overview the role of the elements involved in the process, e.g., resources or personal and possible controlling capabilities. In such situations the advanced knowledge about the elements and the interdependency between them is helpful and should be provided by the software application.

(iii) Experts have the profound knowledge about both elements involved in the process with all its attributes and interdependency between them as significant in the situation under evaluation and control as those will be significant under another condition. Experts have the deep tacit understanding across area of application and can use if ever the software application for testing the own intuition or tacit knowledge.

On the other side the knowledge of the system users will be considered without its subdivision in parts like process knowledge, IT knowledge, domain knowledge, general knowledge, etc. Developers of the application and their knowledge will be considered only with respect to professional knowledge in the application domain. The knowledge about the system development, programming languages and tools, implementation of inference engine or knowledge base(s) will be considered as preliminary owned at needed level of expertise. So the only perspective of the knowledge investigation by developers of the software application is their learning capabilities and based on this growing knowledge in the application domain. As mentioned in introduction this is very important for the quality of the application.

\section{Learning capabilities of users}

Well known statement says that intelligent information systems will be developed not for the experts but based on their expertise in the field. Responsible for this is the knowledge gap among the involved personals, as shown in Fig. 3.

The existence of this knowledge gap between the majority and experts is the basis for realization of intelligent IT systems. If this gap exists through the years then there is the basis for implementation of intelligent application. Developers try to place the knowledge capacities of the software application closer to the upper horizontal line. The goal here is the empowerment of the users without deep knowledge $-20 \%$ line- toward the expert capability which is marked by the upper horizontal line around $80 \%$ on the knowledge axis.

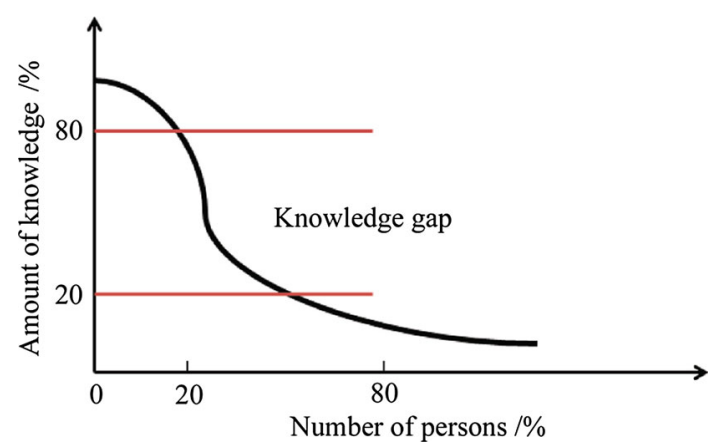

Fig. 3 The knowledge gap

The project team has to analyze this or similar statement and to derive the specific goals and perspectives especially regarding the professional knowledge to be presented and utilized in an intelligent application. The crucial question in current research is how the outline in Fig. 3 changes with time. How many weeks, months the system will work with the same interface and interaction metaphor, and the amount and quality of professional knowledge implemented and visualized in it?

Necessary changes of interaction with system (first of all it relates to the design and realization of the user interface) can be caused through the lessons learned by users and developers of the application. All three groups of users mentioned above have their specific knowledge imperative and learning scope.

The novices will first learn the descriptions of all included elements and needs at this stage of the application utilization rather the declarative knowledge about the process and elements to be controlled. After few months' experience with the application they will be familiar with the application handling and can cope with more complex structures and interdependency. This is the first milestone for review of the interaction concept and for change of the professional knowledge to be used there. The number of such milestones cannot be fixed-it depends on the complexity of the process to be controlled and involved elements as well as learning capability of its users.

Competent users have the knowledge about the elements so they are interested in knowledge about interdependency between the process elements and their utilization within the controlling activities. Corresponding changes of the application with respect to this group will be also necessary after few months. It depends on individual capabilities of competent users-tendentially it will take place later then the explained before the first redesign and update the application for novices. As in previous case it is connected with the complexity of the process to be controlled and its appropriate implementation in the software application. 
Experts as mentioned above have rather small demand to extend their professional knowledge because of expertise they just own. They prove their intuition and tacit knowledge in one or another situation and/or check the knowledge base that will be used for interaction with novices and competent users. Additionally they can extend the knowledge base or test its quality and value for particular case. The last might be done based on some new scenarios of applications utilized for different processes to be controlled.

Occasional users are not intended in owning the professional knowledge about the process to be controlled and details of software application. They can therefore be well supplied by operating instructions presented by IT system to be strictly fulfilled. Some kind of exhaustive explanation and presentation of appropriate knowledge on the user interface is obviously not required.

Vital importance for the software application has the learning capabilities of its developers. Taking into account that the particular application is the one from the set of solutions they have realized or will realize next and knowing that their expertise area is the IT system or application development, one can state that developers are belonging to novices in respect to professional knowledge regarding the process to be controlled.

\section{Process controlling}

The primary interest of management in every enterprise and also in every business process is the efficient and effective handling in every situation $[2-4,11]$. This is the decisive point of the software applications to be installed and utilized in different processes. Efficiency of the controlling is related to the time, effort or cost needed to make a decision and manage the process. Effectiveness of process controlling is linked to resources used to meet the business requirements defined by management.

In respect to human resources, this means that the process controlling realized by competent users or novices can be improved by assistance through the software application. Regarding the time, process controlling executed by experts will be more efficient than those carried out by others. However it will bring significantly more cost. Because of their expertise in the field, the experts will be able to fulfil more requirements defined for the process than novices or competent users.

To improve the process controlling and make it more efficient and effective, all software applications implemented to support the users have to have appropriate scope of flexibility fitted to every user with his individual skill in every possible situation. Such fitness function is outlined in

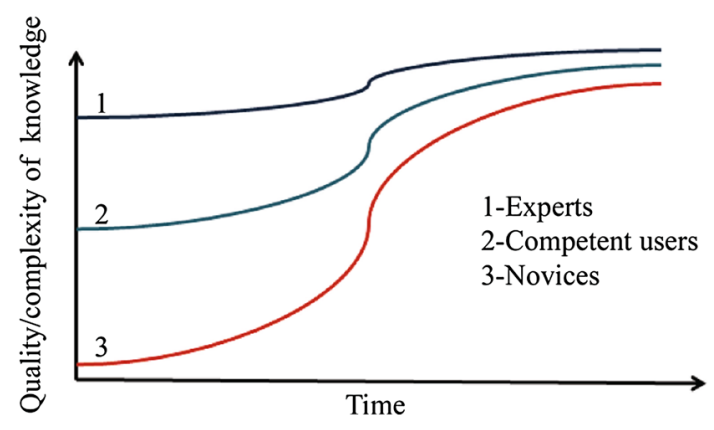

Fig. 4 Dynamic of knowledge quality

Fig. 4 in respect to user group, quality/complexity of professional knowledge and time.

Implementation of the corresponding knowledge bases in the software application will allow and support the flexibility of its utilisation. It seems to become obligatory in every intelligent software application not only to implement several interaction concepts for different groups of users but also to expand this by dynamisation of them in time.

To realize an efficient management of the knowledge bases applied in system to serve the specific person acting at certain point of business process, it is highly recommended to implement the special software module for its controlling. Such software module or tool is primarily focused on the process to be controlled, corresponding knowledge bases, interaction metaphor, and implemented user interface. It works up the available knowledge about business process, and user matches the currently applied interface with this knowledge. The flexibility of interaction, which means describing data dynamically when achieving this way, will provide better service for user and so improve the quality of the system and efficiency of the process controlling in business.

\section{User interface and knowledge visualization}

In this section the concept for knowledge visualization and design of user interface based on discussed above approach of flexible dynamic knowledge is presented. The user interface is selected because of its key role among other components of intelligent software application concerning the realization of the interaction in the system. The process controlling on the other side, e.g., in manufacturing is focused on quick decisions and acting. Therefore the visualization of process activities, proposals for the decisions/actions, and corresponding professional knowledge play a crucial role in realization as the users interface as the software application itself $[12,13]$. 


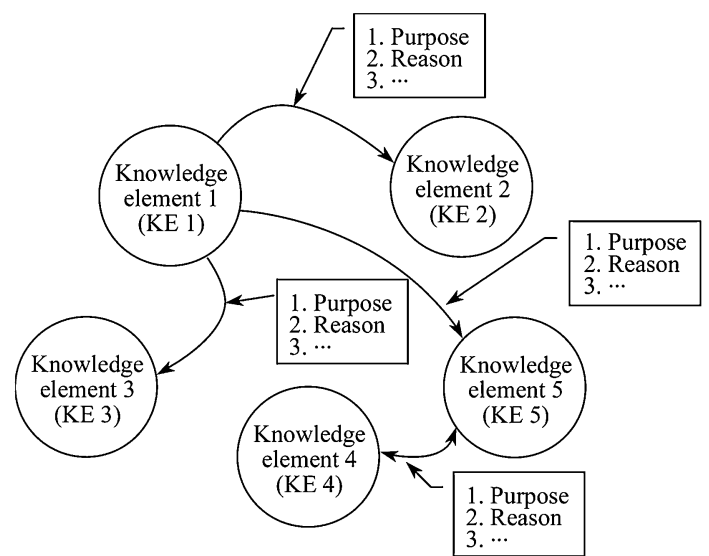

Fig. 5 Purpose driven knowledge visualization

In the following concept our previously developed approach for purpose driven knowledge visualization [14, 15] presented in Fig. 5 will be utilized. This approach will be extended and adapted to application for process controlling. According to previous discussion, this extension contains as declarative as procedural knowledge along three dimensions:

(i) Process knowledge specification with respect to every single activity has to be stored in appropriate knowledge bases in relation to goals to be achieved, resources available and user's classification.

(ii) Knowledge about resources necessary to master the process in every step is the second dimension of knowledge stored. This knowledge should be specified in connection to the knowledge about the process but also to the professional knowledge and experience of individual user.

(iii) User's knowledge in the domain and in the systems handling is the second dimension to be regarded. It also covers learning capabilities with respect to routine tasks and new situation to be managed.

Important aspect of the proposed visualization is the chance to follow different ways to the goal. This corresponds to real situations in the business where some alternatives could often be chosen by managing the process. Some activities can be realized parallelly to each other and some of them could be executed in different orders. Based on the analysis of the available resources, capacities and capabilities of the personnel involved the software application can propose to optimize the process. In this way the staff will learn how the decisions are made during controlling the activities based on the best practice. According to the above discussion it will be possible to test

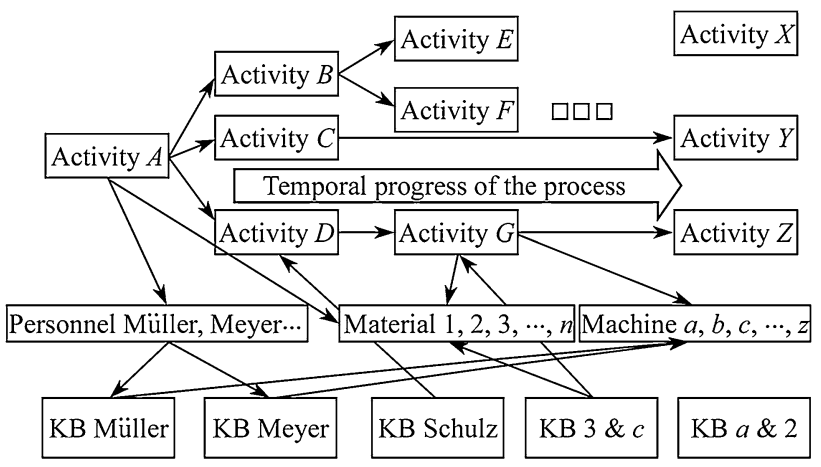

Fig. 6 Dynamic knowledge for process controlling

some new approaches or alternatives. Experience gathered within the test of such alternatives will be evaluated and stored in the knowledge base related to process knowledge. The extended approach for the dynamic knowledge utilization in the intelligent process of controlling solutions is presented in Fig. 6.

Obviously it is impossible to represent the whole range of interdependency in the outline like this. Presented in the upper part of Fig. 6 temporal progress of the process contains the hierarchies of single activity. They can be executed by available resources presented in the middle part. One of the resources included is the staff presented by knowledge bases at the bottom of the outline. Every employee has different qualifications, capabilities, and responsibilities. So for instance Muller can realize the activity $A$ because of his skill to operate the machine $b$ needed to manufacture the material 3 in the selected action. In addition to this rather simple sequence there exist a number of influential aspects which have to be respected by planning and controlling every activity in the process. Decisive element for the controlling as mentioned before is the professional knowledge of responsible person and his experience.

Every activity in the structure outlined contains its own logic and influence factors. Other structures, e.g., personal organization and established responsibilities can be important for one or another decision following controlling of the process.

In the software application for process controlling it is important to focus the interaction on the selected activity. On the user interface there have to be presented involved resources and interdependency between them like in the mentioned purpose driven knowledge visualization approach. To make the controlling efficient, it is necessary to analyze the skill and professional knowledge of management. The knowledge bases in the software applications have to contain different knowledge about the process, resources available or included and intellectual capabilities and skill of involved personnel. 


\section{Conclusions}

In the presented researches, the usage of the professional knowledge in intelligent software applications for process controlling is investigated. It is especially important for the realization of the user interaction and interface design. Because of the complexity and flexibility of the controlling problem, it is proposed to integrate several knowledge bases in the applications which contain all important knowledge for controlling and decision making. The complexity of the interdependency between the elements does not allow the representation of the whole structure, therefore it will be proposed to visualize the only selected activity(ies) in the user interface. This enables the presentation of different involved resources used and staff involved in the activity to be fulfilled. In addition, this presentation will be changed according to the temporal growing of skill by staff or because of the application of new materials or machines.

\section{References}

1. Laudon KC, Laudon JP (2014) Management information systems. Prentice Hall International, Upper Saddle River
2. Dumas M, La Rosa M, Mendling J et al (2013) Fundamentals of business process management. Springer, Berlin

3. Weske M (2012) Business process management, 2nd edn. Springer, Berlin

4. Vom Brocke J, Rosemann M (2010) Handbook on business process management 1,2 nd edn. Springer, Berlin

5. Gadatsch A (2012) Grundkurs Geschäftsprozess-management. Springer, Berlin

6. Thomas D (1993) Process innovation-reengineering work through information technology. Harvard Business School Press, Boston

7. Rowold J (2013) Human resource management. Springer, Berlin

8. Hackos JT, Redish J (1998) User and task analysis for interface design. Wiley, New York

9. Galitz WO (2007) The essential guide to user interface design, 3rd edn. Wiley, Indianapolis

10. Dreyfus HL, Dreyfus SE (1986) Mind over machine: the power of human intuition and expertise in the age of the computer. Basil Blackwell, Oxford

11. Komus A (2011) BPM best practice. Springer, Berlin

12. Keller T, Tergan SO (2005) Knowledge and information visualization. Springer, Berlin

13. Ursyn A (2013) Perceptions of knowledge visualization. IGI Global, Hershey

14. Bodrow W, Magalashvili V (2009) Knowledge visualization in IT-based discovery learning. Commun Cognit 42(1\&2):105-116

15. Bodrow W (2012) Knowledge visualization in academia and business. In: Proceedings of the MASS conference (MASS 2012), Shanghai, pp 1-4 